\title{
Journal of Biomedical Science Reviewer Acknowledgement 2015
}

Wen-Chang Chang

\section{Contributing reviewers}

The editors of Journal of Biomedical Science would like to thank all our reviewers who have contributed to the journal in Volume 22 (2015).

L Adorini

USA

\section{A Ahmad \\ Canada}

Miho Akimoto

Japan

Ola Ali

Egypt

Jalal Alizadeh

Austria

Paulo Amaral

UK

Doblin Anak Sandai

Malaysia

Katsuyuki Aozasa

Japan

Nadezda Apostolova

Spain

Bahram Arjmandi

USA

\section{N Azevedo \\ Portugal}

Li-Yuan Bai

Taiwan

Andrey Barkhash

Russia

\author{
S Barr \\ Canada \\ F Barreto dos Santos \\ Brazil
}

Alexander Bayden

USA

Andrea Belin

Sweden

Thorsten Berger

Canada

Ben Berkhout

Netherlands

Robbert Berkhout

Netherlands

Claudia Biondi

Argentina

Ernst Bomhard

Germany

Emanuela Bostjancic

Slovenia

\section{A Bretscher \\ USA}

Giacomina Brunetti

Italy

Chandana Buddhala

USA
D Canals

USA

A Carnero

Spain

Tai-Lung Cha

Taiwan

D Chan

Hong Kong

Julie Y.H. Chan

Taiwan

Nei-Li Chan

Taiwan

Samuel H.H. Chan

Taiwan

Kai-Ping Chang

Taiwan

Nan-Shan Chang

Taiwan

Jer-Wei Chang

Taiwan

Jang-Yang Chang

Taiwan

Long-Sen Chang

Taiwan

Ming-Fu Chang

Taiwan 


\begin{tabular}{|c|c|c|}
\hline $\begin{array}{l}\text { Rui Chang } \\
\text { USA }\end{array}$ & $\begin{array}{l}\text { Yi-Rong Chen } \\
\text { Taiwan }\end{array}$ & $\begin{array}{l}\text { Cristina Clement } \\
\text { USA }\end{array}$ \\
\hline Jang-Yang Chang & Wei-Yu Chen & Clementina Cocuzza \\
\hline Taiwan & Taiwan & Italy \\
\hline Tsuchung Chang & Yen-Chou Chen & Dawn Coletta \\
\hline Taiwan & Taiwan & USA \\
\hline Wei-Chiao Chang & Yi-Jen Chen & G Coombs \\
\hline Taiwan & Taiwan & Australia \\
\hline Yu-Sun Chang & Yi-Rong Chen & Clark Cullen \\
\hline Taiwan & Taiwan & USA \\
\hline Zee-Fen Chang & I Cherepanova & Guoli Dai \\
\hline Taiwan & USA & USA \\
\hline Lee-Young Chau & Ya-Hui Chi & Niann-Tzyy Dai \\
\hline Taiwan & Taiwan & Taiwan \\
\hline Wassim Chehadeh & Ing-Ming Chiu & P Debre \\
\hline Kuwait & Taiwan & France \\
\hline Yi-Jen Chen & Jeng-Jiann Chiu & Jeffrey Deiuliis \\
\hline Taiwan & Taiwan & USA \\
\hline Bing-Chang Chen & Il Kyu Cho & C Dissous \\
\hline Taiwan & USA & France \\
\hline Ben-Kuen Chen & S Choi & Sipho Dlamini \\
\hline Taiwan & Korea, South & South Africa \\
\hline Chien-Chang Chen & Yu-Ting Chou & W Duan \\
\hline Taiwan & Taiwan & USA \\
\hline Chang-Han Chen & Sin-Tak Chu & E Eugenin \\
\hline Taiwan & Taiwan & USA \\
\hline Jau-Nian Chen & Lee-Ming Chuang & Hua-Chang Fang \\
\hline USA & Taiwan & Taiwan \\
\hline Yung-Ming Chen & PC Chuang & Kang Fang \\
\hline Taiwan & Taiwan & Taiwan \\
\hline Ching-Chow Chen & Shuang-En Chuang & Kleber Farias \\
\hline Taiwan & Taiwan & Brazil \\
\hline Guobing Chen & Huai-hu Chuang & P Fei \\
\hline USA & Taiwan & USA \\
\hline Kuen-Feng Chen & Yao-Chung Chuang & Gregg Fields \\
\hline Taiwan & Taiwan & USA \\
\hline Pei-Jer Chen & Yung-Jen Chuang & Jorge Filmus \\
\hline Taiwan & Taiwan & Canada \\
\hline Ruey-Hwa Chen & R Chun & Janet Flatley \\
\hline Taiwan & USA & UK \\
\hline Chang-Han Chen & Bon-chu Chung & J Frisch \\
\hline Taiwan & Taiwan & Germany \\
\hline Ya-Wen Chen & Chih-Pin Chuu & Wen-Mei Fu \\
\hline Taiwan & Taiwan & Taiwan \\
\hline
\end{tabular}


Bruno Gaillet

Canada

David Gewirtz

USA

Payam Gharibani

USA

Anuradha Ghosh

USA

Tommaso Giani

Italy

Stephen Ginsberg

USA

N Gleicher

USA

M Golczak

USA

M Gotte

Germany

M Gottschalk

Canada

J Greenberger

USA

R Guabirada

UK

Ana Paula Guedes Frazzon

Brazil

Jih-Hwa Guh

Taiwan

K Gurvinder

Australia

K Gurvinder

Australia

B Haagmans

Netherlands

Charles Halsted

USA

Stephen Hare

UK

John Harwood

UK

L Hathaway

Switzerland

M Henry-Stanley

USA
Edward Hitti

Saudi Arabia

S Hladky

UK

Tingjun Hou

China

George Hsiao

Taiwan

Pei-Wen Hsiao

Taiwan

Chung-Bao Hsieh

Taiwan

Hsiu Mei Hsieh

Taiwan

Patrick Hsieh

Taiwan

Po-Shiuan Hsieh

Taiwan

Sung-Tsang Hsieh

Taiwan

Chin Hsu

Taiwan

Hsin-Ling Hsu

Taiwan

Ching-Sheng Hsu

Taiwan

Kuei-Sen Hsu

Taiwan

Yu-Juei Hsu

Taiwan

Hsin-Ling Hsu

Taiwan

Yi-Ping Hsueh

Taiwan

Teh-Min Hu

Taiwan

Eagle Yi-Kung Huang

Taiwan

F Huang

USA

Chung-Feng Huang

Taiwan

Yi-Tsau Huang

Taiwan
Jing-Ding Huang

Taiwan

Kun-Lun Huang

Taiwan

Li-Rung Huang

Taiwan

Shih-Ming Huang

Taiwan

Tze-Sing Huang

Taiwan

Tur-Fu Huang

Taiwan

Menggui Huang

USA

Xupei Huang

USA

Y Huang

Taiwan

Yishuian Huang

Taiwan

Ying-Zu Huang

Taiwan

Dueng-Yuan Hueng

Taiwan

Wen-Chun Hung

Taiwan

Li-Man Hung

Taiwan

Yi-Jen Hung

Taiwan

Liang-Yi Hung

Taiwan

Gero Hutter

Germany

John Imig

USA

Nobue Itasaki

UK

Jukka Jernvall

Finland

Lan Jiang

USA

Shih-Sheng Jiang

USA 
Zheng Jiang

USA

Jeffrey Jones

USA

Yuh-Shan Jou

Taiwan

Chi-Chang Juan

Taiwan

Suh-Hang Juo

Taiwan

S Jurriaans

Netherlands

Shinji Kamada

Japan

S Kamiya

Japan

J Kanczler

UK

J Kang

Korea, South

Reiji Kannagi

Taiwan

Jia-Horng Kao

Taiwan

Murat Kasap

Turkey

S Kent

Australia

H Kida

Japan

Ushio Kikkawa

Japan

K Kim

USA

M Knight

USA

Jan Korbel

Germany

John Kung

Taiwan

Jean-Cheng Kuo

Taiwan

Yu-Min Kuo

Taiwan
Ching-Chuan Kuo

Taiwan

Kuo-wang Tsai

Taiwan

Dortet Laurent

France

Hsuan-Shu Lee

Taiwan

T-C Lee

Taiwan

Wei-Chia Lee

Taiwan

Huei Lee

Taiwan

Hwan Young Lee

Korea, South

Ming-Shyue Lee

Taiwan

Shou-Dong Lee

Taiwan

Hsin-Chen Lee

Taiwan

Yi-Hsuan Lee

Taiwan

Tzong-Shyuan Lee

Taiwan

Wen-Sen Lee

Taiwan

Yi-Hsuan Lee

Taiwan

Yi-Chia Lee

Taiwan

Guey-Jen Lee-Chen

Taiwan

Steve Leu

Taiwan

Euphemia Leung

New Zealand

C Li

Taiwan

Quanhai Li

China

Hua-Jung Li

Taiwan
Chih-Chia Liang

Taiwan

Shu-Mei Liang

Taiwan

Chun-Yen Lin

Taiwan

Feng-Yen Lin

Taiwan

Kwang-Huei Lin

Taiwan

Kai-Ti Lin

Taiwan

Kuo-I Lin

Taiwan

Shuei-Liong Lin

Taiwan

Ya-Wen Lin

Taiwan

Jen-der Lin

Taiwan

Kai-Ti Lin

Taiwan

ShuWha Lin

Taiwan

Su-Fang Lin

Taiwan

Yi-Ling Lin

Taiwan

Wen-Chang Lin

Taiwan

Wan-Wan Lin

Taiwan

Yee-Shin Lin

Taiwan

M Linnebank

Germany

Jyh-Ming Liou

Taiwan

Jun-Yang Liou

Taiwan

Hsiao-Sheng Liu

Taiwan

Chun-Jen Liu

Taiwan 
Fu-Tong Liu

USA

Chen-Hua Liu

Taiwan

Wang-Ta Liu

Taiwan

Shing-Hwa Liu

Taiwan

Shih-Tung Liu

Taiwan

Shing-Hwa Liu

Taiwan

Ko-Jiunn Liu

Taiwan

Y Liu

USA

Z Liu

USA

Jeng-Fan Lo

Taiwan

J Louboutin

Jamaica

Sheng-Nan Lu

Taiwan

Shao-Chun Lu

Taiwan

Chih-Cherng Lu

Taiwan

Ida Gjervold Lunde

USA

Marthandan Mahalingam

USA

M Marazzi

Italy

T. Masami

Japan

Isao Matsuura

Taiwan

N Micale

Italy

Abhisek Mitra

USA

Jigar Modi

USA
David Morris

USA

P Muller

Switzerland

E Nakayama

Japan

Britto Nathan

USA

Melyssa Negri

Brazil

A Niaj

Poland

B Nikolajczyk

USA

N Nunthaboot

Thailand

Chunliu Pan

China

Liuliu Pan

USA

Li-Heng Pao

Taiwan

J Park

Korea, South

M Parsons

Australia

S Pechine

France

Hwei-Ling Peng

Taiwan

A Perl

USA

Peter Bradding

UK

A Ping

USA

John Pitman

USA

Hemant Poudyal

Japan

Spyros Pournaras

Greece

Howard Prentice

USA
Prabhakar Putheti

USA

M Rashid

Pakistan

Mickae Rialland

France

Gabriel Rinaldi

UK

Harrison Roger

USA

J Routy

Canada

U Ruffing

Germany

Chiranjeevi Sandi

UK

Michael Schmidt

USA

Erhard Seifried

Germany

Chia-Ning Shen

Taiwan

Ethan Shevach

USA

Sheau-Yann Shieh

Taiwan

Jin-Yuan Shih

Taiwan

Shin-Ru Shih

Taiwan

Venkatakrishna Shyamala India

Jia-Fwu Shyu

Taiwan

Kou-Gi Shyu

Taiwan

Rajvir Singh

USA

Po-Chi Soo

Taiwan

K Steinmetzer

Germany

Tung-Hung Su

Taiwan 
Huaichang Sun

China

Cheuk-Kwan Sun

Taiwan

Nao Suzuki

Japan

Man-Sun Sy

USA

Ming-Hong Tai

Taiwan

You-Lin Tain

Taiwan

Xiaoyu Tain

Hong Kong

Pao-Luh Tao

Taiwan

Rui Tao

USA

Woan-Yuh Tarn

Taiwan

Omid Teymournejad

Iran

Chenxi Tian

USA

N Topley

UK

Kenneth Tsai

USA

Kun-Chih Tsai

Taiwan

Hsien-Chun Tseng

Taiwan

Che-Se Tung

Taiwan

Alexandar Tzankov

Switzerland

Natsuo Ueda

Japan

Haluk Vahaboglu

Turkey

M Varecha

Czech Republic

E Vicenzi

Italy
KS Vishwanatha

USA

Hsian-Jenn Wang

Taiwan

Jaw-Yuan Wang

Taiwan

Jia-Yi Wang

Taiwan

Danny Ling Wang

Taiwan

Lu-Hai Wang

Taiwan

Ju-Ming Wang

Taiwan

Feng-Sheng Wang

Taiwan

Jin-Town Wang

Taiwan

Feng-Sheng Wang

Taiwan

Jin-Town Wang

Taiwan

Ju-Ming Wang

Taiwan

Yi-Ching Wang

Taiwan

Yun Wang

Taiwan

Yau-Huei Wei

Taiwan

Jianning Wei

USA

Shu-Chen Wei

Taiwan

Wei Wei

USA

Dirk Westermann

Germany

Dionna Williams

USA

Martin Witzenrath

Germany

Chin-Chen Wu

Taiwan
Han-Chung Wu

Taiwan

Jen-Leih Wu

Taiwan

Jinhua Wu

USA

Bin-Nan Wu

Taiwan

Yi-Chun Wu

Taiwan

Hua-Lin Wu

Taiwan

Kay LH Wu

Taiwan

T.-C. Wu

USA

Kou-Juey Wu

Taiwan

Betty Wu-Hsieh

Taiwan

Xiwen Xiong

USA

Jun Xu

USA

Yemin Xu

USA

Xiaoyu Xue

USA

Chuen-Mao Yang

Taiwan

Chih-Wei Yang

Taiwan

HC Yang

Taiwan

Jenq-Lin Yang

Taiwan

MH Yang

Taiwan

Ruey-Bing Yang

Taiwan

Rong-Sen Yang

Taiwan

Sung-Sen Yang

Taiwan 
Shang-Hsun Yang

Taiwan

Muh-Hwa Yang

Taiwan

Wei-Shiung Yang

Taiwan

San Nan Yang

Taiwan

Yang Yang

USA

Lin Ye

USA

Chau-Ting Yeh

Taiwan

Wei-Lan Yeh

Taiwan
B. Linju Yen

Taiwan

Jiin-Cherng Yen

Taiwan

Mao-Hsiung Yen

Taiwan

Shaw-Fang Yet

Taiwan

Hon-Kan Yip

Taiwan

Ming-Lung Yu

Taiwan

Linda Chia-Hui Yu

Taiwan

Wei-Hsuan Yu

Taiwan
Dah-Shyong Yu

Taiwan

Chiou-Hwa Yuh

Taiwan

Shaoyun Zang

USA

Ulrich Zanger

Germany

Xi Zhan

USA

Jing Zhang

USA

D Zhang

USA 